\title{
A multilevel fast spectral domain algorithm for electromagnetic analysis of infinite periodic arrays with large unit cells
}

\author{
T. F. Eibert \\ Institute of Radio Frequency Technology, University of Stuttgart, Germany
}

\begin{abstract}
A multilevel fast spectral domain algorithm (MLFSDA) is introduced for the efficient evaluation of the matrix vector products due to the boundary integral (BI) operator within a hybrid finite element - BI (FEBI) method for the analysis of infinite periodic arrays. The MLFSDA utilizes the diagonalization property of the spectral domain (SD) BI representation and handles the large numbers of Floquet modes required for large (with respect to wavelength) periodic unit cells by similar hierarchical techniques as applied in the multilevel fast multipole method/algorithm (MLFMM/MLFMA). With the capability of the MLFSDA to handle very large periodic unit cells, it becomes possible to model finite antennas and scatterers with the infinite periodic array model. For a cavity-backed antenna element and for a semi-finite array of 4 cavity-backed antenna elements in the finite direction, the dependence of the input impedances on the unit cell sizes is investigated and it is found that array resonances disappear for reasonably large unit cell dimensions. Finally, a semi-finite array of antipodal Vivaldi antenna elements is considered and simulation results for infinite periodic, finite, and semi-finite array configurations are compared to measured data.
\end{abstract}

\section{Introduction}

The infinite periodic array model is still an important approach for analysis and design of large finite arrays. With periodic excitation, the field solution in the array becomes also periodic and Floquet's theorem can be employed to reduce the computational domain down to a single array element (unit cell), thus significantly speeding up analysis and reducing memory requirements. In the context of integral equation (IE) formulations, periodic Green's functions are constructed, where especially spectral domain (SD) repre-

Correspondence to: T. F. Eibert (eibert@ihf.uni-stuttgart.de) sentations amenable to multilayered configurations are used (Mittra, 1988; Aroudaki et al., 1995). Arbitrarily inhomogeneos array elements can be modelled by the hybrid finite element - boundary integral (FEBI) technique (McGrath and Pyati, 1994; Lucas and Fontana, 1995; Eibert et al., 1999, 2000, 2003). Most approaches work with simple half-space Green's functions in SD (McGrath and Pyati, 1994; Lucas and Fontana, 1995), whereas a spatial domain Green's function computed via the Ewald transformation is used in Eibert et al. (1999). Also, the SD half-space Green's function has been extended to multilayer configurations above and below the FE mesh in Eibert et al. (2003). The approach in Eibert et al. $(2000,2003)$ evaluates the SD BI very efficiently by a so-called fast spectral domain algorithm (FSDA), but the approach works efficiently only for relatively small unit cell dimensions (with respect to wavelength), since the necessary Floquet mode numbers increase with the size of the pertinent unit cells.

In non-periodic IE and FEBI approaches, the use of fast integral solvers such as the fast multipole method (FMM) and its multilevel versions (MLFMM/MLFMA) (Chew et al., 2001) or the adaptive integral method (AIM) (Bleszynski et al., 1996) (similarly pre-corrected FFT methods Gedney et al., 2003) have become standard over the past years. Periodic IE and FEBI formulations can also be accelerated by fast integral methods. However, large problems with large unit cells require to work with large numbers of Floquet modes (or other types of series terms in spatial domain or mixed spatial/spectral representations (Ewald transformation)) and this leads to considerably increasing computational effort, even with the use of conventional fast integral methods.

In FSDA (Eibert et al., 2000), it was already realized that the standard SD formulation leads to diagonalization of the pertinent IE and BI operators. In this paper, it is shown that the standard SD formulation can be cast in an FMM-like representation. Thus, FSDA and multilevel FSDA (MLFSDA) approaches can be derived by similar techniques

Published by Copernicus GmbH on behalf of the URSI Landesausschuss in der Bundesrepublik Deutschland e.V. 


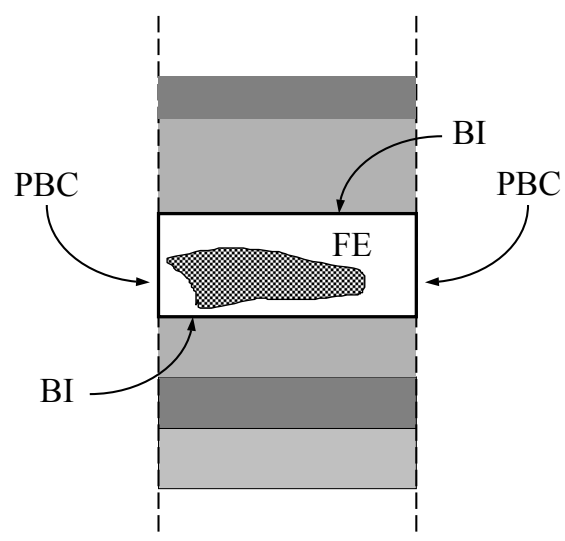

Fig. 1. Cross section of array unit cell with indication of BI and periodic boundary condition (PBC) surfaces.

as employed for FMM and MLFMM/MLFMA. Compared to MLFMM/MLFMA, MLFSDA does not need any nearcoupling corrections and all interactions are computed on the coarsest level with only one group. For the considered periodic problems, the summation over the Floquet wavenumbers represents an exact integration rule and also the translation operator evolves directly from the SD representation. In this paper, MLFSDA is employed to accelerate a FEBI technique working with tetrahedral volume and triangular BI elements.

With MLFSDA, unit cells with dimensions of many wavelengths can be efficiently handled. Thus, it is possible to model multilayer FSS with dissimilar periodicities by the supercell approach, where a period common to all individual layers is defined and several array elements in the various layers are placed in the computational unit cell. This approach is probably not as efficient as the cascading technique in Ma et al. (2005), but has increased modelling flexibility.

With the capability to handle large unit cells, it becomes possible to place entire finite configurations in a periodic unit cell, which is large enough to decouple the periodic images. Thus, the advantages of the periodic modelling approach (Floquet mode series instead of SD integral) can also be used to compute finite configurations such as finite antenna arrays.

The paper is organized as follows. After presenting some basic equations, the MLFSDA technique and the modelling of finite and semi-finite configurations are discussed. In the results section, the transmission coefficient of an 8-layer FSS with dissimilar periodicities is computed and compared to measured data. For the examples of a cavity-backed patch antenna element and a corresponding quadruple patch array, it is investigated which unit cell sizes are necessary to decouple the periodic images in finite and semi-finite configurations, respectively. Finally, a semi-finite array of antipodal Vivaldi antenna elements is investigated.

\section{Formulation}

\subsection{Hybrid FEBI formulation}

The hybrid FEBI formulation for infinite periodic array configurations employing Floquet's theorem to reduce the problem to a single unit cell of the array (see Fig. 1) can be found in many references such as McGrath and Pyati (1994); Lucas and Fontana (1995); Eibert et al. (1999, 2003). The periodicity in the $x y$-plane is defined by the lattice vectors $\boldsymbol{\rho}_{a}, \boldsymbol{\rho}_{b}$ via the shifting relation

$\boldsymbol{\rho}_{m n}=m \boldsymbol{\rho}_{a}+n \boldsymbol{\rho}_{b}, \quad \boldsymbol{r}=\boldsymbol{\rho}+z \hat{z}=x \hat{\boldsymbol{x}}+y \hat{\boldsymbol{y}}+z \hat{z}$,

where $m$ and $n$ are integers. Assuming the periodicity conditions given in Eibert et al. (1999), electromagnetic analysis can be carried out by enforcing stationarity of a standard FE functional in terms of the electric field intensity $\boldsymbol{E}$ with BI termination for the FE portion of the unit cell, shown in Fig. 1.

\subsection{Multilevel fast spectral domain algorithm}

In a standard implementation of the hybrid FEBI method, the BI contribution results in fully populated matrix blocks for the unknowns in the top and bottom BI surfaces and therefore it is desirable to evaluate the BI expression in an alternative manner. It is well known that the standard SD formulation diagonalizes the BI operator and this property was already utilized for the formulation of an FSDA in Eibert et al. (2000). In this section, an MLFSDA is derived which allows very fast evaluation of the BI contributions during the computation of matrix-vector products in an iterative equation solver, even for large unit cells.

We start with the SD representation of the spatial Green's function in the BI

$$
\begin{aligned}
\overline{\mathbf{G}}_{\mathrm{p}}\left(\boldsymbol{\rho}, \boldsymbol{\rho}^{\prime}\right) & =\iint_{k_{x}} \int_{k_{y}} \tilde{\overline{\mathbf{G}}}_{\mathrm{p}}\left(k_{x}, k_{y}\right) \\
& \times \mathrm{e}^{-j k_{x}\left(x-x^{\prime}\right)} \mathrm{e}^{-j k_{y}\left(y-y^{\prime}\right)} d k_{x} d k_{y},
\end{aligned}
$$

where

$$
\tilde{\overline{\mathbf{G}}}_{\mathrm{p}}\left(k_{x}, k_{y}\right)=\frac{1}{A} \sum_{p=-\infty}^{\infty} \sum_{q=-\infty}^{\infty} \tilde{\overline{\mathbf{G}}}\left(k_{x}, k_{y}\right) \delta\left(\boldsymbol{k}_{t}-\boldsymbol{k}_{t p q}\right)
$$

in the case of an infinite periodic array problem with

$$
\begin{aligned}
A & =\left|\boldsymbol{\rho}_{a} \times \boldsymbol{\rho}_{b}\right|, \boldsymbol{k}_{t}=k_{x} \hat{x}+k_{y} \hat{y}, \boldsymbol{k}_{t p q} \\
& =\boldsymbol{k}_{t 00}+\frac{2 \pi}{A}\left[p\left(\boldsymbol{\rho}_{b} \times \hat{z}\right)+q\left(\hat{z} \times \boldsymbol{\rho}_{a}\right)\right],
\end{aligned}
$$

and $\tilde{\overline{\mathbf{G}}}$ the corresponding non-periodic SD Green's function (see Eibert et al., 1999, 2003) and introduce the decomposition

$\boldsymbol{\rho}-\boldsymbol{\rho}^{\prime}=\left(\boldsymbol{\rho}-\boldsymbol{\rho}_{g}\right)+\left(\boldsymbol{\rho}_{g}-\boldsymbol{\rho}_{g}^{\prime}\right)-\left(\boldsymbol{\rho}^{\prime}-\boldsymbol{\rho}_{g}^{\prime}\right)$ 
based on a regular grouping of the basis functions in the BI surfaces. $\rho_{g}$ is the group center close to observation point $\rho$ and $\rho_{g}^{\prime}$ is the group center close to the source point $\boldsymbol{\rho}^{\prime}$. Substituting these expressions into the discretized BI contribution (e.g. see Eibert et al., 1999) gives

$$
\begin{aligned}
& \iint_{S} \boldsymbol{b}_{m}(\boldsymbol{\rho}) \cdot \iint_{S} \overline{\mathbf{G}}_{\mathrm{p}}\left(\boldsymbol{\rho}, \boldsymbol{\rho}^{\prime}\right) \cdot \boldsymbol{b}_{n}\left(\boldsymbol{\rho}^{\prime}\right) d s^{\prime} d s \\
& =\iint_{k_{x}} \int_{k_{y}} \tilde{\boldsymbol{b}}_{m}^{*}\left(k_{x}, k_{y}\right) \cdot \overline{\mathbf{T}}_{m g, n g}\left(k_{x}, k_{y}\right) \cdot \tilde{\boldsymbol{b}}_{n}\left(k_{x}, k_{y}\right) d k_{x} d k_{y}
\end{aligned}
$$

with

$\tilde{\boldsymbol{b}}_{n}\left(k_{x}, k_{y}\right)=\iint_{S} \boldsymbol{b}_{n}(\boldsymbol{\rho}) \mathrm{e}^{j k_{x}\left(x-x_{n g}\right)} \mathrm{e}^{j k_{y}\left(y-y_{n g}\right)} d s$

and the corresponding translation operator

$$
\begin{aligned}
\overline{\mathbf{T}}_{m g, n g}\left(k_{x}, k_{y}\right) & =\tilde{\overline{\mathbf{G}}}_{\mathrm{p}}\left(k_{x}, k_{y}\right) \\
& \times \mathrm{e}^{-j k_{x}\left(x_{m g}-x_{n g}\right)} \mathrm{e}^{-j k_{y}\left(y_{m g}-y_{n g}\right)} .
\end{aligned}
$$

Also, $\boldsymbol{b}_{n}$ are the usual Rao-Wilton-Glisson basis functions used to descretize the BI. Equation (5) has the same structure as diagonalized FMM representations (Chew et al., 2001), except that the FMM integration over the Ewald sphere is replaced by SD lateral wavenumber integration, i.e. a truncated Floquet mode series in the case of the considered infinite periodic problem (see Eq, (3)). Thus, by applying the same principles as done in FMM or MLFMM/MLFMA, fast and multilevel fast spectral domain algorithms (FSDAs and MLFSDAs) can be realized to evaluate the BI. However, some important differences are observed. Most importantly, the translation operator in Eq. (5) is known analytically and the whole representation Eq. (5) is exact for all interactions, including near- and self-terms. Moreover, for infinite periodic problems the integration rule for the computation of the SD integral in Eq. (5) is also exactly given by the Floquet mode representation of the periodic Green's function (see Eq. (3)). Consequently, a particular error analysis is not necessary for FSDA and MLFSDA, since the algorithms give the same results as conventional SD implementations. In MLFSDA, we must only guarantee that the required interpolation and anterpolation steps are carried out without introducing additional errors.

FSDA has already been presented in Eibert et al. (2000). Due to the exact validity of Eq. (5), this algorithm does not work with any grouping and computes all interactions among all basis functions at once. This algorithm is well suited for periodic problems with small unit cells, however, becomes inefficient for large unit cells, where an increasing number of Floquet modes is needed. In MLFSDA, large unit cells are covered by a regular square grid of groups and a hierarchical grouping structure is introduced. Since FSDA and MLFSDA do not suffer from low-frequency break-down, the size of the

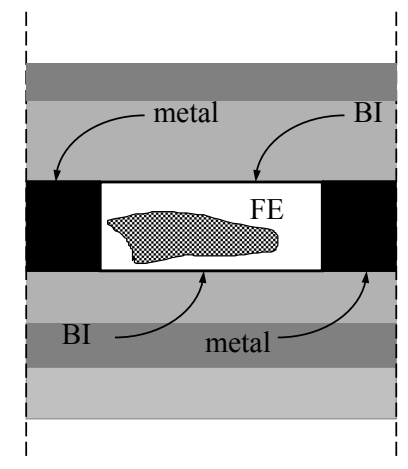

Fig. 2. Cross section of array unit cell for finite array modelling with indication of BI surfaces.

groups on the finest level can be chosen on the same order as the sizes of the discretization subdomains. On higher levels, lower level groups are combined until there is only one group on the coarsest level $L$. All interactions according to Eq. (5) are computed for the single group on the coarsest level, where the SD sample density is chosen according to the size of this group. One level below, every other sample is left out and so on until the finest level is reached. The sampling density on the lower levels is determined by the sizes of the groups on these levels. The sample densities on the various levels are determined according to the Shannon sampling theorem, where sufficient oversampling must be provided for the interpolation and anterpolation procedures to work.

\subsection{Computation of finite and semi-finite configurations by increasing the infinite array period}

With the MLFSDA, it is possible to compute periodic array problems with large unit cells. For many array problems, this is not important, since the period is often on the order of a half wavelength. However, large unit cells are required if multilayer FSSs with dissimilar periodicities shall be computed by the supercell approach and we can even place an entire finite or semi-finite configuration in one unit cell. Since the influence of the periodic unit cell images on the finite configuration must be small in this case, the period in the computational periodic array model must be chosen very large and a model according to Fig. 2 evolves from the original model in Fig. 1. Due to the use of a Green's function with metallic backing in the BI, the finite array in the unit cell is embedded in a metallic plate with thickness according to the height of the FE domain. In practical computations, we found that the influence of the periodic images on the finite array vanishes for reasonably large periodic array periods, especially if little (negligible for finite configuration) losses are assumed in some of the material layers modelled in the BI. 


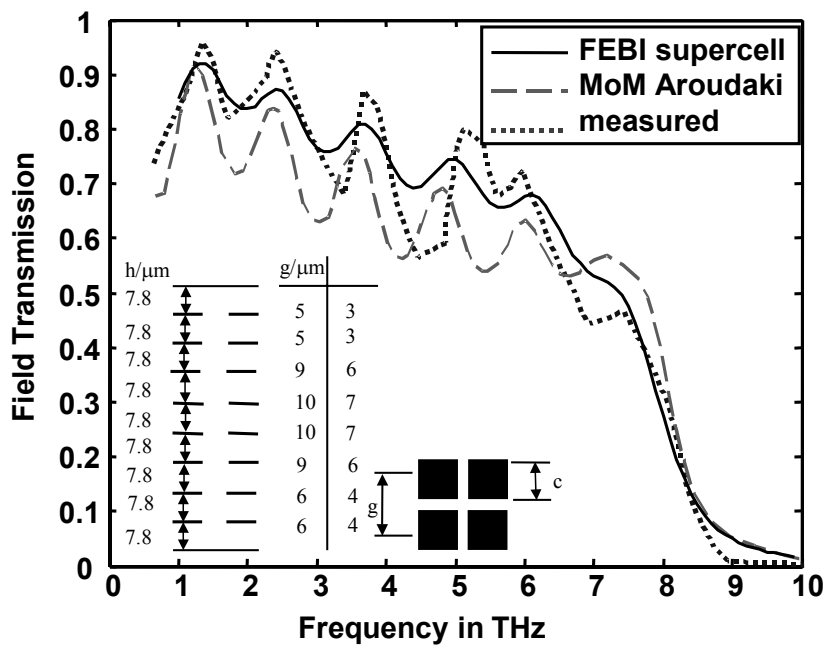

Fig. 3. Near-infrared 8-layer low-pass FSS with dissimilar periodicities, $\varepsilon_{r}=2.31, \tan \delta=0.035$ in FEBI computations, incidence angle $30^{\circ}$ with respect to normal, power average of TE and TM.

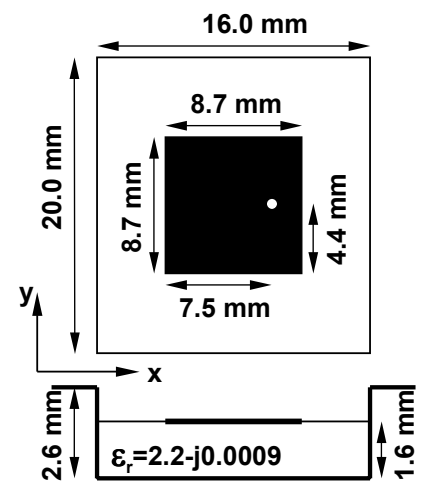

Fig. 4. Cavity-backed patch antenna element.

\section{Results}

The first example is the near-infrared 8-layer FSS with dissimilar periodicities depicted in Fig. 3 and already considered in Aroudaki et al. (1995). For a valid infinite periodic model, the supercell approach must be applied for this problem. The dimensions of the used (smallest) supercell are $90 \mu \mathrm{m}$ by $90 \mu \mathrm{m}$ resulting in up to 18 by 18 patch elements in the upper two layers.

Unfortunately, material losses and fabrication tolerances are not very well known for this FSS. Our FEBI-MLFSDA computations were performed for a dielectric substrate with $\varepsilon_{r}=2.31-j 0.08$. A truncated Floquet mode series ranging from -7 to 7 was used for the representation of the basis function radiation patterns on the finest level and the Floquet mode series representing the Green's function on the coarsest level ranged from -53 to 53 . The discretization model consisted of about 3.6 Mio volume unknowns including 20223 BI unknowns. The utilized RAM was about 2.8 GByte in-

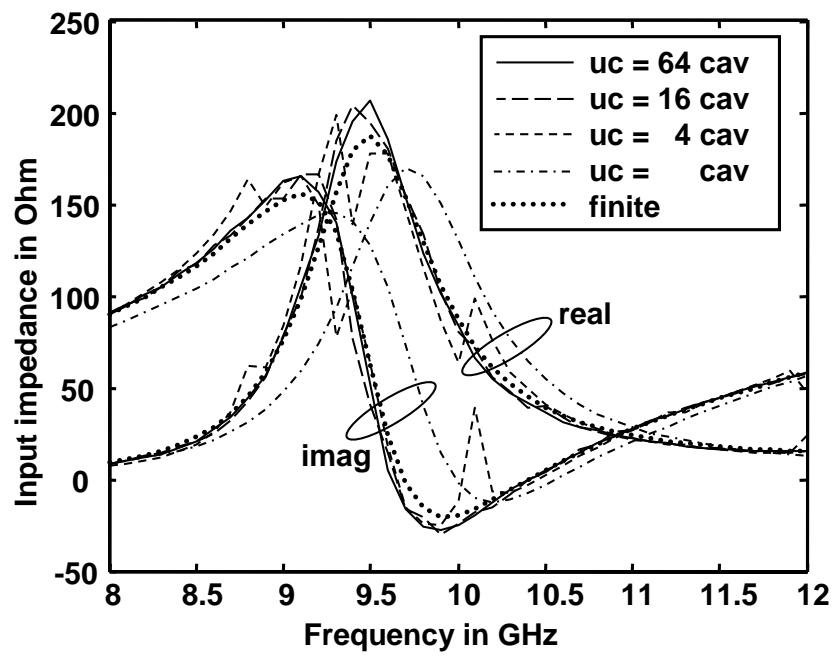

Fig. 5. Input impedance of cavity-backed patch antenna element according to Fig. 4 for infinite periodic array configurations with increasing unit cell dimensions.

cluding about 1.8 GByte for storing the search vectors in the GMRES solver. Field transmission results are given in Fig. 3 and compared to measured results from Aroudaki et al. (1995) and approximate MoM results from Aroudaki et al. (1995), obtained using only one patch in every layer. Especially near the passband edge of the low-pass, our FEBIMLFSDA supercell results capture the measured behavior more accurately.

The next problem is intended to demonstrate the feasibility of the approach discussed in Sect. 2.3 for the treatment of finite configurations. A simple cavity-backed patch antenna element excited by a probe current as depicted in Fig. 4 is considered. Reference computations were carried out by a 3-D hybrid FEBI code for finite problems, where the cavitybacked patch antenna element was placed in a thick finite metallic ground plane of size $160 \mathrm{~mm}$ by $200 \mathrm{~mm}$ with height $5 \mathrm{~mm}$. Infinite periodic array computations with the FEBIMLFSDA code were performed for equally increasing unit cell sizes in the two array dimensions in order to reduce the coupling between the unit cell patch and its periodic images. The input impedance results in Fig. 5 contain clearly visible array resonances for unit cell sidelengths (uc) of 4 and 16 times the cavity sidelengths (cav), but the curves for $\mathrm{uc}=64 \mathrm{cav}$ do not show anymore obstruction by array resonances and agree pretty well with the finite ground plane reference results. When the unit cell has the same size as the cavity, the input impedance is also smooth but shows a noticeable frequency shift compared to the finite ground plane results. Due to anterpolation of the translation operator in the setup phase, the sample density required on the coarsest level could be kept very small ( -6 to +6$)$, no matter how large the unit cell was chosen.

Next, a finite by infinite array consisting of 4 patch ele- 


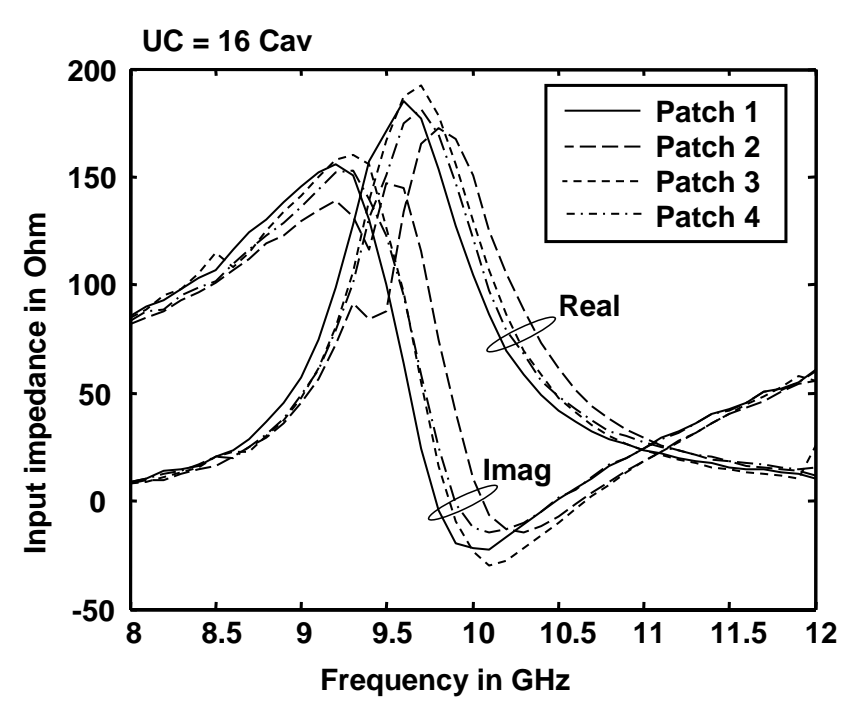

Fig. 6. Input impedances of finite by infinite patch array (4 elements in finite direction) consisting of patch elements according to Fig. 4 obtained with infinite periodic array model with a unit cell 16 times larger than the size of the array in the finite direction.

ments according to Fig. 4 in the finite direction is considered. In the infinite $y$ direction, the patch elements are separated by metallic walls, whereas no metallic walls are present in the finite $x$ direction. Figs. 6 and 7 show the input impedances of the 4 patch elements within the unit cell chosen for the infinite periodic FEBI-MLFSDA computations. The results in Fig. 6 computed with a periodic unit cell sidelength (uc) of 16 times the size of the array in the finite direction are clearly influenced by array resonances due to the periodic images in the computational model. However, after increasing the sidelength of the periodic unit cell to 512 times the array size in the finite direction, no more array resonances can be observed in the input impedance results in Fig. 7.

The final example is a semi-finite phased-array antenna of antipodal Vivaldi elements as illustrated in Fig. 8 and discussed in Wilden et al. (2004). The array consists of 8 equally-fed elements on one substrate in the finite direction and up to 256 active elements in the other direction (periodic boundaries indicated by dashed lines in the figure). The advantage of the antipodal Vivaldi elements is that they can be fed by microstrip lines. However, a $180^{\circ}$ phase shift is required between neighboring feed ports. The fabricated antenna elements are fed by 1 to 8 power dividers, where the necessary phase shifts are realized by rat-race couplers. In our simulations, each Vivaldi element was fed by a short piece of coaxial line (excited by impressed line currents in the FE mesh) and forward and backward travelling waves on the coax line were extracted by the matrix pencil method in order to determine the pertinent $S$ parameters. First simulations were performed for a double element of the antipodal Vivaldi radiators. Figure 9 shows simulation results for a free-standing double element computed by a hybrid FEBI

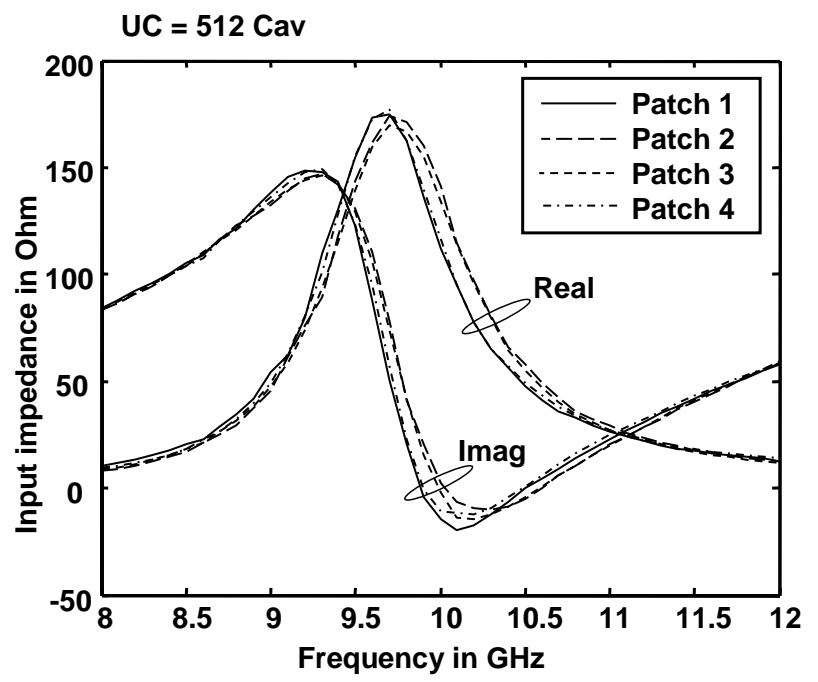

Fig. 7. Input impedances of finite by infinite patch array (4 elements in finite direction) consisting of patch elements according to Fig. 4 obtained with infinite periodic array model with a unit cell 512 times larger than the size of the array in the finite direction.

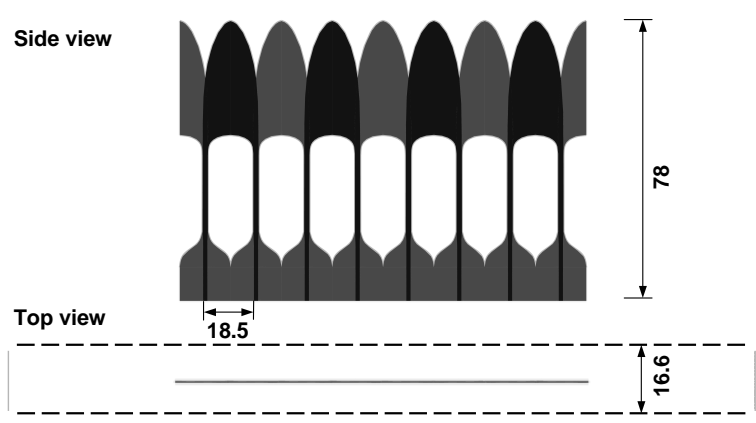

Fig. 8. Front (black) and rear (dark grey) side metallizations of 8element column of antipodal Vivaldi elements. The dashed lines in the top view represent the periodic boundaries, the through lines at left and right sides indicate the electric boundaries in the FE domain for the semi-finite simulations (dimensions in $\mathrm{mm}$, substrate: Rogers Duroid, $\varepsilon_{r}=2.2,0.5 \mathrm{~mm}$ thick).

code for finite configurations and compares it to the passive reflection coefficient directly measured at the input of a middle element of an 8-element column, where the remaining elements were terminated by $50 \Omega$ plugs. The agreement of simulated and mesured results is very reasonable.

Figure 10 compares simulated infinite periodic results (obtained by FEBI-MLFSDA according to periodic array dimensions as indicated in Fig. 8) and measured active reflection results. The measured active reflection coefficient was found for a middle element of a finite array of 9 8-element columns by considering the coupling to all elements in the finite array. Given the relatively small size of the measured array, it is remarkable that the simulated array resonance peak at about $10 \mathrm{GHz}$ is also found in the measured data. Simulated array resonances at other frequencies are not that obvious in the measured results. 


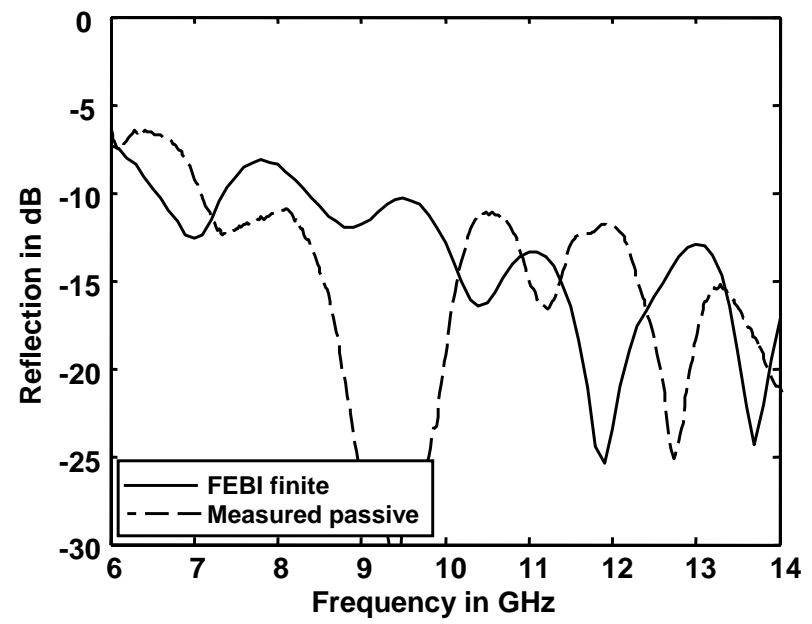

Fig. 9. Simulated and measured reflection coefficients for antipodal Vivaldi antenna element.

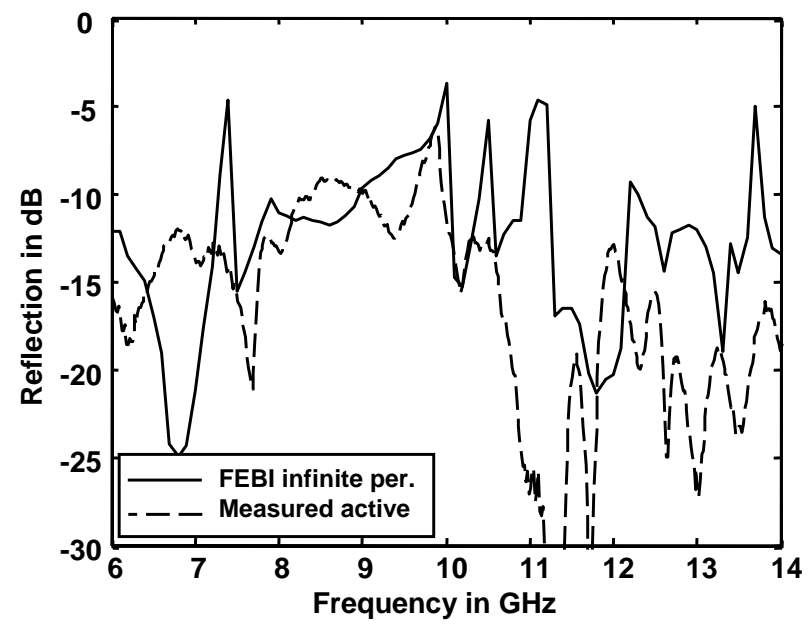

Fig. 10. Simulated and measured reflection coefficients for antipodal Vivaldi antenna array.

Further FEBI-MLFSDA simulations were performed for the semi-finite array configuration (see Fig. 8). The computed reflection coefficients of the 8 elements in the column were combined according to the 1 to 8 power divider used to feed the fabricated antenna. The resulting active reflection coefficient (FEBI semi-finite (sum)) is shown in Fig. 11 and compared to measured results obtained at the input port of the power divider (Measured active (sum)). Again, the coupling to 9 columns was considered to determine measured active results. For comparison, also the simulated infinite periodic element results are included. Interesting to note is, that the simulated semi-finite sum results show considerably weaker array resonances than the infinite periodic element results. In the measured active sum results determined at the input port of the power divider, almost no array resonances are found and the active reflection coefficient is below $-10 \mathrm{~dB}$

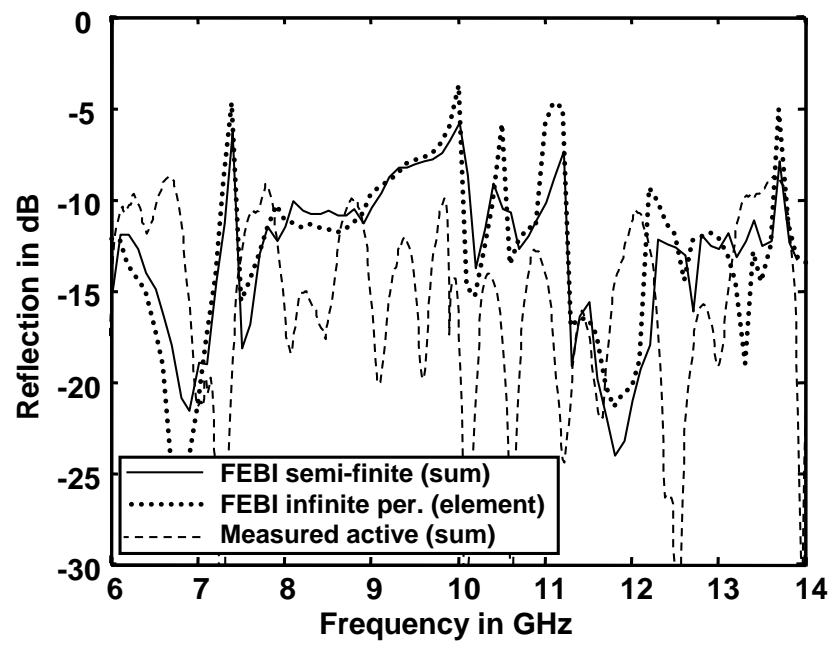

Fig. 11. Simulated and measured reflection coefficients for antipodal Vivaldi antenna array.

in most parts of the considered frequency range. The averaging among the 8 array elements in the finite array direction obviously suppresses the array resonances.

\section{Conclusions}

The hybrid finite element - boundary integral (FEBI) method for the analysis of infinite periodic arrays was extended by a multilevel fast spectral domain algorithm (MLFSDA) which accelerates the matrix vector product evaluation for the BI operator. The MLFSDA utilizes the diagonalization property of the spectral domain BI representation and employs similar multilevel procedures as applied in the multilevel fast multipole method/algorithm (MLFMM/MLFMA). With MLFSDA, large (with respect to wavelength) periodic unit cells can be treated by the FEBI method, which are for instance necessary for the modelling of multilayer frequency selective surfaces (FSSs) with dissimilar periodicities by the supercell method. Moreover, it is now possible to solve finite electromagnetic antenna and scattering problems by the infinite periodic approach und utilize its simplified evaluation of the BI operator (Floquet mode series instead of spectral integral). The finite antenna or scatterer is placed in the periodic unit cell and the dimensions of the unit cell are chosen large enough to reduce coupling between the periodic images to a negligible level. For a finite and a semi-finite cavity-backed patch antenna problem, the necessary sizes of the computational unit cells were studied. Finally, a semi-finite array of antipodal Vivaldi antenna elements was investigated. Since the presented FEBI method works with mutilayered Green's functions including metallic backing in the BI surfaces, the approach is very well suited for antennas and scatterers embedded in a thick metallic plate and not so well for freestanding configurations. However, the MLFSDA has the po- 
tential so speed-up the evaluation of various infinite periodic integral equation operators (e.g. electric field integral equation for planar circuits and antennas in multilayered media).

\section{References}

Mittra, R., Chan, C. H., and Cwik, T.: Techniques for Analyzing Frequency Selective Surfaces - A Review, Proc. IEEE 76, 1593-1615, 1988.

Aroudaki, H., Hansen, V., Gemünd, H.-P., and Kreysa, E.: Analysis of Low-Pass Filters Consisting of Multiple Stacked FSS's of Different Periodicities with Applications in the Submillimeter Radioastronomy, IEEE Trans. Antennas Propagat., 43, 14861491, 1995.

McGrath, D. T. and Pyati, V. P.: Phased Array Antenna Analysis with the Hybrid Finite Element Method, IEEE Trans. Antennas Propagat., 42, 1625-1630, 1994.

Lucas, E. W. and Fontana, T. W.: A 3-D Hybrid Finite Element/Boundary Element Method for the Unified Radiation and Scattering Analysis of General Infinite Periodic Arrays, IEEE Trans. Antennas Propagat., 43, 145-153, 1995.

Eibert, T. F., Volakis, J. L., Wilton, D. R., and Jackson, D. R.: Hybrid FE/BI Modeling of 3-D Doubly Periodic Structures Utilizing Triangular Prismatic Elements and a MPIE Formulation Accelerated by the Ewald Transformation, IEEE Trans. Antennas Propagat., 47, 843-850, 1999.
Eibert, T. F. and Volakis, J. L.: Fast Spectral Domain Algorithm for Hybrid Finite Element/Boundary Integral Modeling of Doubly Periodic Structures, IEE Proceedings Microwaves, Antennas Propagat., 147, 329-334, 2000.

Eibert, T. F, Erdemli, Y. E., and Volakis, J. L.: Hybrid Finite Elemente - Fast Spectral Domain Multilayer Boundary Integral Modeling of Doubly Periodic Structures, IEEE Trans. Antennas Propagat., 51, 2517-2520, 2003.

Chew, W. C., Jin, J.-M., Michielssen, E., and Song, J.: Fast and Efficient Algorithms in Computational Electromagnetics, Boston: Artech House, 2001.

Bleszynski, E., Bleszynski, M., and Jaroszewicz, T.: AIM: Adaptive Integral Method Compression Algorithm for Solving LargeScale Electromagnetic Scattering and Radiation Problems, Radio Science, 31, 1225-1251, 1996.

Gedney, S. D., Zhu, A., Tang, W.-H., Liu, G., and Petre, P.: A fast, high-order quadrature sampled pre-corrected FFT for electromagnetic scattering, Microwave Opt. Technology Letters, 36, 343-349, 2003.

Ma, J.-F., Mittra, R., and Huang, N. T.: Analysis of Multiple FSS Screens of Unequal Periodicity Using an Efficient Cascading Technique, IEEE Trans. Antennas Propagat., 53, 1401-1414, 2005.

Wilden, H., Poppelreuter, B., Saalmann, O., Brenner, A., and Ender, J.: Design and Realisation of the PAMIR Antenna Frontend, EUSAR, 493-496, 2004. 